\title{
Meta
}

Journal des traducteurs

Translators' Journal

\section{Essai d'étude comparative des locutions et termes d'un glossaire technique plurilingue tendant à dégager certaines tendances générales, caractéristiques de chaque langue}

\section{Jacques Goetschalckx}

Volume 18, numéro 1-2, mars 1973

Actes du deuxième colloque international de linguistique et de traduction. Montréal, 4-7 octobre 1972

URI : https://id.erudit.org/iderudit/002929ar

DOI : https://doi.org/10.7202/002929ar

Aller au sommaire du numéro

Éditeur(s)

Les Presses de l'Université de Montréal

ISSN

0026-0452 (imprimé)

1492-1421 (numérique)

Découvrir la revue

Citer cet article

Goetschalckx, J. (1973). Essai d'étude comparative des locutions et termes d'un glossaire technique plurilingue tendant à dégager certaines tendances générales, caractéristiques de chaque langue. Meta, 18(1-2), 261-267. https://doi.org/10.7202/002929ar 


\title{
Essai d'étude comparative des locutions et termes d'un glossaire technique plurilingue
}

\author{
TENDANT À DÉGAGER CERTAINES TENDANCES GÉNÉRALES \\ CARACTÉRISTIQUES DE CHAQUE LANGUE
}

Dans la préface de leur Stylistique comparée de l'anglais et du français, MM. Vinay et Darbelnet relatent qu'en parcourant l'autoroute de New York à Montréal leur curiosité fut éveillée par des panneaux de signalisation qui ne laisssaient pas de faire surgir quelques problèmes de langue. C'est un peu de la même manière, c'est-à-dire au fil de nombreuses traductions, de centaines et centaines de pages, que j'ai buté sur des problèmes de traduction bien sûr, mais aussi sur des problèmes de langue et même de civilisation. Je crois d'ailleurs que cette démarche pragmatique est celle de la plupart des traducteurs de ma génération.

Le linguiste en général ou le traducteur en particulier est ainsi fait qu'il ne peut plus voir ou entendre un terme ou une phrase sans se demander comment cela se dirait dans une autre langue. En revanche, lorsqu'il rencontre une formule peu orthodoxe, il devient soupçonneux et cherche l'influence contaminante d'une langue étrangère par la faute d'un traducteur peu respectueux du génie de la langue dans laquelle il traduit.

Il est certain que dans le langage général ce qui est qualifié de propre au génie de la langue procède tout autant du génie du peuple qui parle cette langue. En revanche, la langue à son tour influe sur la manière de penser et la manière d'être de tous ceux qui ont $\ll b u » c e t t e$ langue avec le lait maternel.

C'est ainsi que le Frainçais, qui aime sortir, marche dans la rue, il s'y sent chez lui. En revanche, le Néerlandais, qui est un homme d'intérieur, marche sur la rue. Il se voit marcher sur le trottoir mais il ne se sent pas pris par la rue. Lui, il garde ses distances. Il est vrai qu'en français le mot trottoir suscite des associations d'idées d'un tout autre genre, liées à une branche d'activités très ancienne. Mais ne soyez pas trop surpris non plus si une brave ménagère belge, soucieuse de l'hygiène publique et de l'état de propreté de sa rue, vous confie qu'elle fait le trottoir le samedi : Honni soit qui mal y pense. Nous voyons ainsi que les possibilités de méprise sont multiples et pleines de risques.

Mais ces tendances fondamentales semblent se manifester également dans le langage technique. Pensons par exemple à l'inscription No entry. À première vue cela ne paraît pas tellement technique, mais puisqu'il s'agit d'une indication utile cette expression relève de la signalisation et prend de ce fait un caractère technique. 
Prenons donc ce No entry : le Français, fidèle à l'esprit de Montesquieu, voit les choses sous l'angle juridique «défense d'entrer». L'Anglo-Saxon, foncièrement fonctionnaliste, déclare purement et simplement que ce passage n'est pas une entrée. Le Français en est tout aussi conscient sans doute mais, inventeur du système D., il pourrait néanmoins être tenté d'utiliser ce passage comme entrée tant qu'il n'y a pas l'interdiction formelle.

Mais avant de m'engager davantage dans le développement de ce thème, je voudrais vous expliquer quelle est la matière première sur laquelle j'ai travaillé pour faire le présent exposé. Il s'agit de contextes prélevés dans des documents techniques ayant servi à la composition de glossaires. Ceux qui ont assisté au congrès organisé il y a deux ans ont entenđu l'exposé de M. Bachrach sur la méthode terminologique désignée par le sigle DICAUTOM. Pour ceux qui n'y étaient pas, je me permettrai d'exposer brièvement les principes de cette méthode.

DICAUTOM tend à établir un corpus multilingue et phraséologique comprenant des termes techniques relatifs à des domaines très variés. Partant du principe que le mot ne prend toute sa signification que dans un contexte naturel, nous préférons juxtaposer des phrases en laissant au traducteur ou à d'autres usagers le soin d'en extraire les termes ou les expressions qui les intéressent. Ces phrases ne sont pas des constructions artificielles mais des phrases authentiques prélevées sur des documents techniques originaux. En procédant de la même manière pour les différentes langues de travail, nous pouvons à la longue trouver des phrases qui - moyennant certains aménagements ou par amalgame si vous voulez sont à considérer comme équivalentes. Pour l'essentiel, les phrases présentées ont donc toutes été trouvées telles quelles dans des documents originaux traitant de problèmes bien délimités.

Pourquoi avons-nous adopté cette formule? Le raison principale est que nous voulions composer ainsi un dictionnaire automatique - de-là le sigle DICAUTOM qui pourrait être consulté directement. En clair, cela veut dire que les phrases-exemples extraites par les terminologues sont enregistrées dans la mémoire d'un ordinateur. Le système est conçu de telle manière que la question peut être posée sous forme d'une phrase ou d'un membre de phrase.

En réponse, la machine fournit des phrases-exemples comprenant les éléments de la phrase-question. Pour éviter des avalanches de phrases pour certains mots clés assez fréquents, le programme prévoit un calcul de concordance grâce auquel la réponse se concentre sur les phrases-exemples les plus significatives. Ce n'est donc pas un simple système d'archivage automatisé, comme disait M. Quemada hier matin. Au contraire, en travaillant par association, la machine peut même donner plus qu'on n'en a mis dedans !

Pour tirer quelque enseignement vraiment valable de l'analyse de textes techniques, il faudrait évidemment que l'étude porte sur des dizaines de milliers de phrases. Néanmoins, l'expérience acquise, surtout au cours des huit dernières années, me permet de formuler certaines conclusions, bien qu'il n'y ait jamais eu, du moins jusqu'à présent, d'analyse systématique de toutes les phrases traitées. 
C'est au cours de ces travaux d'analyse de textes techniques et par la comparaison des formes adoptées tans les différentes langues pour exprimer la même notion, la même opération, le même phénomène, que j'ai été frappé par la spécificité de certaines structures et que j'ai commencé à me demander si ces différences répondaient à certaines règles.

Il est bien connu que dans la langue générale ces différences plus ou moins caractéristiques existent. En effet, l'ouvrage de MM. Vinay et Darbelnet le montre amplement. Mais on aurait pu croire que dans le langage technique, où il s'agit surtout de choses purement objectives et directement saisissables, il n'en soit pas ainsi ou du moins pas dans la même mesure. Dans la langue générale, on exprime des pensées ou des sentiments. Il s'ensuit que là l'engagement personnel est total. Il est donc logique que les penchants propres à chaque individu, à chaque race ou à chaque peuple se manifestent dans la façon de s'exprimer. Or, l'analyse de textes techniques qui a été faite dans les conditions que je vous ai esquissées donne à penser que dans le domaine technique également cette tendance fondamentale subsiste et cela se traduit par des termes et des expressions qui reflètent, à mon sentiment, le caractère propre de ceux qui les emploient.

Examinons quelques exemples de textes techniques anglais et français. En parlant du traitement d'un cordon de soudure, le texte français parle de « meulage de la surépaisseur », l'anglais parle de plane down. Nous constatons donc que le français indique l'opération à effectuer cependant que l'anglais, en ajoutant le mot down, pense tout de suite au résultat à atteindre. Dans un autre texte traitant de problèmes d'érosion, nous trouvons en français «la perte de matière n'est pas accélérée proportionnellement à la vitesse d'écoulement ». Dans un équivalent anglais, la notion «perte de matière» se traduit par rate of removal. Ici c'est donc le cas contraire, l'anglais parle du processus removal, le français indique le résultat «perte de matière ». Cependant dans les deux cas nous voyons que le français a une tendance à l'abstraction «meulage», "perte de matière », tandis que l'anglais est plus près de la réalité en parlant de plane down et removal.

Comme point de référence en matière de température, le français retient la «température ambiante». Or, nous savons que l'anglais préfère à la notion plus ou moins abstraite de «ambiant» la notion très nette et très précise de room temperature qui donne un cadre très précis et nous dirions presque très humain.

Nous trouvons un phénomène analogue dans le domaine des essais de chaudières où le français emploie généralement l'expression «épreuve hydraulique » alors que l'anglais parle sans détours du water pressure test.

De même, en cristallographie, le français parlera de "lacunes dans le réseau cristallographique $\gg$ cependant que l'anglais parlera tout simplement de hole. Dans ce cas également s'oppose au terme très simple et très objectif de hole un vocable qui est beaucoup plus complexe et trahit, à mon sens, à nouveau ce penchant pour l'abstraction puisque "lacune » signifie beaucoup plus qu'un simple vide. La notion de lacune implique tout d'abord que l'on considère l'ensemble et qu'il 
$\mathrm{y}$ a un vide là où il devrait y avoir quelque chose. Toutes ces connotations sont entièrement étrangères au vocable hole.

Un autre phénomène, qui me donne à penser qu'il existe même dans le langage technique certaines différences qui sont caractéristiques du génie des langues française et anglaise, se trouve dans les exemples suivants : en parlant d'un mélange gazeux, le chimiste anglais dit d'un mélange contenant une teneur faible d'une matière active quelconque qu'il s'agit d'un weak mixture. Le chimiste français dira de ce mélange qu'il est «pauvre ». De même le spécialiste d'hygiène industrielle qui s'occupe des problèmes d'empoussiérage définira sa spécialité comme dust control, cependant que son collègue français dira qu'il s'occupe de « la lutte contre les poussières ». La notion weak mixture est une simple constatation, l'adjectif «pauvre » implique que le mélange dont il s'agit devrait, pour bien faire, avoir une teneur plus forte de matière active. Je sais bien que dans certains cas il faut des mélanges pauvres, mais le fait est que le français emploie un terme qui comporte normalement une appréciation qualitative alors que son collègue anglo-saxon emploie une notion plutôt quantitative. Le plus drôle est que dans le cas contraire l'anglais parle aussi de rich mixture.

De même le dust control est une action menée froidement tandis que la notion de «lutte» fait penser à certains mouvements de l'âme qui amènent l'individu à se défendre contre le danger. Cette même tendance se manifeste dans le domaine de la corrosion puisqu'en France on dit d'une certaine corrosion qu'elle est «préférentielle » alors qu'outre-Manche ou outre-Atlantique elle est "selective». Or, il est indiscutable que la préférence est une affaire de cœur et la sélection une affaire de froide intelligence.

Outre cette opposition «abstrait-concret», j'ai donc cru pouvoir déceler une seconde opposition «objectivité-subjectivité ». Mais ne me faites pas dire ce que je n'ai pas dit... je ne crois pas que l'hygiéniste anglo-saxon lutte avec moins d'acharnement contre les méfaits de la poussière que son collègue français, et je n'ai pas dit non plus que la corrosion anglo-saxonne est plus intelligente que la française.

Une troisième ligne de partage serait l'économie. L'anglais est une langue où l'économie est tellement ancrée dans la tradition qu'elle ne pose pas de problèmes. L'anglais peut par exemple très bien parler d' $X$-rays lorsqu'il pense à des clichés radiologiques. En français il existe bien sûr des tendances analogues. Pensons à «construction mécanique » qui vise la construction de pièces mécaniques. Le court-circuitage est moins brutal dans «construction métallique » par exemple, mais il l'est davantage dans «mines métalliques» pour les mines extrayant des minerais qui, par un traitement adéquat, donnent des métaux. Faut-il voir dans cette tendance une manifestation de la réserve britannique? L'Anglais est un homme de peu de mots, le Français est plus volubile.

Il me semble de toute manière que les différences constatées soient caractéristiques et il serait donc intéressant de savoir où elles trouvent leur origine. Il est toujours hasardeux de se lancer dans des conjectures sur les origines profondes de ces différences de conception et pour le faire avec toutes les garanties 
de la science il faudrait que cette étude soit faite par des personnes plus capables que moi, par des sociolinguistes peut-être. Il faudrait en outre qu'elles disposent de l'appareil d'enquete et de recherches nécessaires. Mais il faudrait surtout qu'elles puissent y consacrer une bonne partie de leur temps ce qui n'est malheureusement pas le cas pour moi.

Néanmoins je n'ai pas pu résister à la tentation de lancer quelques idées. Il y a par exemple des mauvaises langues qui disent que si les Scandinaves parlent peu et que lorsqu'ils parlent ils le font pratiquement sans ouvrir la bouche, c'est tout simplement parce qu'il $y$ fait trop froid. Puisque le climat des îles Britanniques n'est pas des plus agréables, on pourrait croire que l'aspect économique de leur langage ait la même cause. Pour les Américains on pourrait dire que c'est dû à l'influence des Quakers qui n'attachent de l'importance qu'à la parole intérieure du Saint-Esprit.

Mais il n'est pas non plus interdit de croire qu'il y ait d'autres raisons pour cette différence de langage. L'histoire de la France a été marquée très tôt par une monarchie très forte et très autoritaire. Sans remonter aux Missi Dominici de Charlemagne dans lesquels on pourrait voir les prédécesseurs de nos distingués inspecteurs des finances, on peut dire que la France dispose depuis très longtemps d'une réelle administration, centralisatrice et traditionaliste. Londres n'a jamais joué le même rôle que Paris et c'est peut-être parce que la Grande-Bretagne tout entière est une île qu'elle n'a pas l'équivalent de l'île-de-France qui, hébergeant le pouvoir central, en a profité pour imposer son dialecte à la France tout entière.

Cette influence prédominante du pouvoir central pourrait expliquer la tendance à l'abstraction, cette tendance à mettre une étiquette sur chaque chose. D'autant que l'étiquette doit être choisie de telle manière qu'elle puisse s'appliquer à toutes les variantes possibles, présentes et à venir, ce qui oblige à recourir à l'abstraction. Devant cette richesse, Albion se trouve complètement démunie puisqu'elle n'a pas de droit codifié ni même une constitution, du moins pas sous cette forme bien nette que l'on connaît en France, surtout depuis qu'un très grand monsieur s'en est occupé.

En revanche, il faut bien reconnaître que sur le plan de l'industrialisation l'Angleterre avait pris une belle avance sur le continent et qu'il est donc assez logique que, devant ces progrès rapides de la technique et de l'industric, il a fallu trouver, à l'instant même pour ainsi dire, une appellation pour des produits et des processus nouveaux, appellation qui, par la force des choses, s'inspirera étroitement des réalités nouvelles.

Celui qui a le temps de prendre un certain recul peut choisir des termes après mûre réflexion, mais il essaiera de le faire en respectant certaines habitudes et certaines traditions. En revanche le mot préexistant est souvent un handicap car il invite trop facilement au calque et semble inhiber l'esprit d'invention. À ce propos, la Banque de mots de Montréal a prouvé récemment qu'il n'en est pas toujours ainsi. Invitée à se prononcer sur l'équivalent français de l'expression shopping spree à l'occasion d'un concours dont la gagnante avait le droit de faire 
pendant un temps donné un shopping spree, la Banque a trouvé «coup de filet». Terme merveilleux qui, s'écartant résolument du terme original, a trouvé une expression qui se raccroche à une notion existante en s'offrant au passage le luxe de jouer sur l'association avec le filet à provisions de la ménagère.

Revenons maintenant à la question de l'économie. Cette tendance à l'économie s'est certainement accélérée dès que le nouveau continent a fait son entrée sur la scène. Puisque M. Taylor est américain, les chercheurs techniciens et ouvriers américains ont connu avant nous ce que le parti communiste français appelle «les cadences infernales». De plus, comme la société américaine est née d'un amalgame de peuples, de langues, de traditions et de civilisations différentes, il a fallu sans doute se limiter à un langage simple fait de mots que chacun connaît pour aboutir à une terminologie qui ne rebute ni l'un ni l'autre.

Privés des bienfaits d'une longue tradition, ils n'en ont pas non plus subi les entraves. Nous constatons d'ailleurs que depuis qu'en France également la productivité est devenue un souci majeur, ce qui fait que tout le monde a dû pousser sur l'accélérateur, les Français comme les autres parlent volontiers en sigles, depuis le P.D.G. jusqu'à l'O.S.

C'est d'ailleurs très curieux de voir comment une tendance contraire se manifeste chez les spécialistes des questions nucléaires. Il faut dire que, là aussi, les Américains avaient déjà une certaine tradition puisqu'ils donnaient toujours de jolis noms de filles aux pires ouragans. Maintenant leurs atomistes reprennent ces habitudes et les collègues français leur emboitent le pas. C'est ainsi que des engins terrifiants sont afflublés de noms pleins de lyrisme comme Masurca ou Rapsodie.

Nous voyons donc que ceux qui vivent dans un monde dur s'efforcent d'en échapper et de survivre grâce à la fraîcheur d'une création où la fantaisie a sa part. C'est une question de biotope.

Il ressort de tout cela qu'il existe certaines tendances plus ou moins caractéristiques des différentes langues. Passer d'une langue à une autre est passer d'un monde à un autre monde. Des générations ont mis leur empreinte sur les formes de la langue. Même une langue de faible diffusion peut être tout un monde parce que ses racines sont profondes. Cela ne veut pas dire évidemment qu'il n'y ait pas et qu'il ne peut pas y avoir d'emprunts d'une langue à une autre, nous le savons bien.

À ce point de vue d'ailleurs les néerlandophones - car ces phones-là existent aussi, il ne faut pas les oublier - les néerlandophones dis-je sont assez fiers d'avoir apporté au français un vocable important — si l'on veut bien admettre que bien s'habiller comme bien manger font partie de la culture - car c'est au néerlandais que le français a emprunté le terme mannequin. Et Dieu sait si le mannequin est intéressant à bien des égards.

Mais pour en revenir à ces tendances générales, je pense qu'il serait très utile d'approfondir cette question. Elle peut avoir son utilité dans la recherche terminologique, dans la recherche des termes existants, mais aussi bien sûr pour 
s'orienter dans la création de termes nouveaux lorsqu'il y a lieu. C'est là qu'il y a tout le mérite du Comité d'études des termes techniques, animé par M. Agron qui, dans la recherche de termes nouveaux, tient compte non seulement du génie de la langue française, mais aussi du génie du citoyen français d'aujourd'hui en chair et en os.

Car, nous avons pu le constater, l'appellation d'une chose ne part pas toujours de l'objet à dénommer. Au contraire, il faut souvent puiser dans un patrimoine culturel très large. Le «coup de filet» en est un exemple éloquent.

En raison des différences fondamentales des structures des différentes langues, il semble bien que pour les glossaires plurilingues c'est la méthode phraséologique qui apporte souvent la meilleure solution aux problèmes du traducteur parce qu'elle juxtapose des morceaux de discours correspondants. Cela postule évidemment que les contextes retenus soient de bons contextes pour reprendre encore un mot de M. Quemada. Il faut des contextes homogènes et significatifs, mettant en relation plusieurs notions associées. Mais cela n'empêche pas que si l'on est génial on peut trouver cette formule lapidaire, percutante, qui est en même temps représentative et caractéristique. Vous en voulez un exemple? C'est «coup de filet ».

J. GoetschalcKX 